\title{
A simple model solution of the suspension bridge problem
}

\author{
by \\ L. F. Stephens, B.Sc., M.E., Ph.D., A.M.I.C.E., M.I.C.E.I., \\ A.M.I.Struct.E.
}

Lecturer, University College, Dublin

Professor T. M. Charlton (The Queen's University of Belfast) suggested that the value of the Paper lay not only in a novel and useful application of one of the simplest techniques of model analysis but also in the evidence it provided in support of the type of linear beam-on-elastic foundation approximation to suspension bridge behaviour suggested by Pugsley. ${ }^{7}$ Thus the Author's models were based on the representation of a suspension bridge as a beam with intermediate discrete linearly elastic supports, the flexibility of each support corresponding to the direct flexibility of the cable (calculated in the manner proposed by Pugsley) and hanger at the relevant point of the span. Quite apart from the approximation inherent in linearization of suspension bridge behaviour, there was then a further approximation in disregarding the 'cross' coefficients of flexibility. From the results presented in the Paper it appeared, however, that this procedure could provide satisfactory estimates of significant maxima.

42. It was believed that similar satisfactory estimates might be obtained readily by calculation using an approximate solution based on the principle of minimum total potential energy. For this purpose the suspension bridge was represented as a beam with intermediate linearly elastic supports precisely as suggested by the Author, and the deflexion $\Delta$ of the deck represented by say, a trigonometrical series which satisfied the boundary conditions, for example:

$$
\Delta=A_{1} \sin \frac{\pi}{l} x+A_{2} \sin \frac{2 \pi}{l} x+A_{3} \sin \frac{3 \pi}{l} x+\ldots . . . .
$$

- where $l$ was the span of the deck and $A_{1}, A_{2}, A_{3}$ etc. are undetermined parameters. This expression for defiexion was then substituted in the expression for the total potential energy of the system:

$$
V=\frac{E}{2} \int_{0}^{l} I\left(\frac{d^{2} \Delta}{d x^{2}}\right)^{2} d x+\frac{1}{2} \sum^{n} b_{i} \Delta_{i}^{2}-\sum^{N} F_{j} \Delta_{f} \quad \text {. . . . }
$$

where $b_{i}$ is the stiffness (reciprocal of the flexibility) of the $i^{\text {th }}$ elastic support and $F_{j}$ is the $j^{\text {th }}$ live load component, and $A_{1}, A_{2}, A_{3}$, etc. calculated by using the condition for stationary potential energy:

$$
\frac{\partial V}{\partial A_{1}}=0 ; \quad \frac{\partial V}{\partial A_{2}}=0 ; \quad \frac{\partial V}{\partial A_{3}}=0 ; \text { etc. }
$$

43. Mr P. Bowen, a research student in the Civil Engineering Dept at Belfast, had obtained rapidly results lying between those of the deflexion theory and model analysis for the Author's example 1 (Fig. 5), using only three terms of the series. ${ }^{7}$ Details of the solution of a problem of this kind are given elsewhere. ${ }^{14}$

44. This method avoided the averaging of the intermediate support stiffnesses which was necessary in applying the beam-on-elastic foundation analogy and which necessitated consequently the use of empirical data for the best results. No recourse to empiricism was necessary for the satisfactory application of the suggested method. 
Incidentally, a better approximation would be obtained by calculating that part of the total potential energy due to the cable in terms of a complete set of stiffness coefficients of the cable. The difficulty here lay in the calculation of the 'cross' coefficients of stiffness by, say, inverting the corresponding flexibility matrix whose constituent flexibility coefficients were easily calculated as proposed by Pugsley. ${ }^{\text {? }}$

45. An alternative approximate approach would consist of representing the bending moment distribution for the deck (or distribution of tension over the hangers) approximately by a series with undetermined parameters which satisfied the boundary conditions of equilibrium, and obtaining the unknowns by the condition for the complementary energy of the structure to be stationary. For this purpose the complementary energy of the cable could be calculated in terms of its readily calculated flexibility coefficients.

46. Professor Charlton was grateful to Dr Stephens for the stimulus which his interesting Paper had provided for further investigation of approximate methods of studying the behaviour of suspension bridges and would appreciate his comments on the methods outlined.

Dr J. Szidarovszky (Ministry of Communication, Budapest) wrote that the model experiment dealt with in the Paper was based on an analogy between numerical analysis of suspension bridges, and of elastically supported beams. This analogy had a deep and fundamental essence. The differential equation of the latter was

and

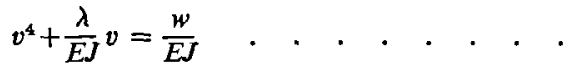

$$
\begin{aligned}
& \lambda=c b
\end{aligned}
$$

where $w$ was distributed load and $\lambda$ a parameter. The homogeneous form of equation (10)

$$
v^{4}+\frac{\lambda}{E J} v=0 \quad . \quad . \quad . \quad . \quad . \quad . \quad . \quad .
$$

48. A non-trivial solution of equation (12) existed only if the $\lambda$ parameter took one of the eigenvalues.

$$
-\lambda_{1}, \lambda_{2}, \ldots,-\lambda_{t} \quad . \quad . \quad . \quad . \quad . \quad . \quad .
$$

where $\left(\lambda_{t}>0\right)$ and the solutions corresponding are the eigenfunctions:

$$
\phi_{1}, \phi_{2}, \ldots, \phi_{i}, \ldots \text {. . . . . . . }
$$

49. If the loading was expressed by a series of the eigenfunctions as:

$$
w=\frac{\lambda}{E J} \sum_{i=1}^{\infty} a_{i} \phi_{i} \text {. . . . . . . . }
$$

the solution of equation (10) was

$$
v=\frac{1}{E J} \sum_{i=1}^{\infty} a_{i} \frac{\lambda}{\lambda+\lambda_{i}} \phi_{i} \quad . \quad . \quad \text {. . . . . . }
$$

50. The differential equation of suspension bridges was:

$$
\begin{array}{cccccccc}
v^{2}-\frac{u}{E J} v=w+H y & . & . & . & . & . & . & . \\
u=H+H_{\mathrm{d}} & . & . & . & . & . & . & .
\end{array} .
$$

where $y$ was the ordinate of cable. The homogeneous form is:

$$
v^{4}-\frac{u}{E J} v=0 \quad \text {. . . . . . . . . }
$$


and so:

$$
v^{4}=\frac{u}{E J} v^{2}
$$

51. From equations (19) and (20)

$$
v^{4}-\left(\frac{u}{E J}\right)^{2} v=0
$$

or

was obtained, where:

$$
v^{4}-\frac{\lambda}{(E J)^{2}} v=0 \quad \text {. . . . . . . . . }
$$

$$
\lambda=u^{2} \quad \text {. . . . . . . . . . }
$$

52. But equation (21) was identical to equation (12), so the eigenfunctions must also be equal. If:

$$
w+H y=\frac{\lambda}{(E J)^{2}} \sum_{i=1}^{\infty} a_{i} \phi_{i} \text {. . . . . . . }
$$

then the solution of equation (17) must be

$$
v=\frac{1}{(E J)^{2}} \sum_{i=1}^{\infty} a_{1} \frac{\lambda}{\lambda+\lambda_{4}} \phi_{i} \quad . \quad . \quad . \quad . \quad . \quad . \quad \text {. }
$$

In the case of simple beam and $E I=$ const (see reference 10)

$$
\phi_{i}=\sin \frac{i \pi x}{l} \quad . \quad . \quad . \quad . \quad . \quad . \quad .
$$

Equation (75) of reference 5 containing smaller effects differed slightly from equation (17); in this case, therefore, the analogy was not exact, but disregarding quantities smaller than the third and higher order, the analogy was close enough to apply.

53. The model experiment presented was very neat and simple, but its chief advantage was not based solely upon the fact that "a simple solution of the problem could be obtained quickly', as the use of tabulated functions was also simple and easy. ${ }^{16.17} \mathrm{~A}$ model analysis, however, neglected more points than a numerical analysis and if the results obtained by both methods were similar it was highly probable that they were reliable.

54. The experiments showed a good agreement with the calculated values in cable forces, and positive moments and hanger forces, but differed in negative moments and hanger forces. The comparison of deflexions was not worked out. Equation (3) showed that the influence of cable extension was disregarded in calculation. In this case the area of the deflexion diagram should be zero. From experiments it was found that the sign hardly changed in cases where the cable extension was large. The Writer proposed to investigate this fact in the hope of producing reasons for the differences mentioned.

The Author, in reply, thanked the contributors for their discussion; by their comments regarding approximate methods for the analysis of suspension bridges they had undoubtedly extended the scope and usefulness of the original Paper.

56. Professor Charlton and Dr Szidarovszky had both demonstrated that the approximate model solution had its counterpart in approximate solutions obtained by calculation; both referred to the representation of the deflected form of the deck as a trigonometrical series satisfying the boundary conditions. This approach, as pointed out by Dr Szidarovszky, had also been used by Timoshenko, ${ }^{10}$ although in the latter case further simplifying assumptions were made which invalidated the solution. 
57. It was encouraging to find that Professor Charlton's results obtained by a series method, using only three terms of the series in conjunction with the principle of minimum total potential energy, agreed substantially with the Author's model results and with other calculated solutions.

58. Professor Charlton correctly drew attention to the fact that the model solution disregarded the cross coefficients of flexibility. It was not, however, true to say that the model solution necessitated the use of empirical data for best results, and it was in this that the method differed from the method proposed by Professor Pugsley. ${ }^{6}$ In the model, the actual hangers were replaced by a reduced number of equivalent hangers at greater spacing, and the results showed that it was normally sufficient to take eight such hangers within the span length to obtain good results.

59. With regard to Dr Szidarovszky's comments, the Author agreed that while the case of a stiffening girder with variable section might be easily analysed if tabulated functions, such as those given in Asplund's paper ${ }^{16}$ were available, some degree of familiarity with such tables was necessary if they were to be used with confidence. The model solution might be used to check solutions obtained by such means.

60. As suggested by Dr Szidarovszky, although the influence of cable extension had been disregarded, the additional deflexion due to this effect could be taken into account in the model. It should be borne in mind, however, that the simple spring analogy could never reproduce exactly the total overall behaviour of a continuous cable, and some error in the portions subject to negative moment must be expected. The Author looked forward to the results of the further investigations proposed by the Contributor.

61. The Paper and the discussion had shown the importance of the equations for cable flexibility derived by Professor Pugsley, ${ }^{7}$ and further work on the application of these equations, and their limitations, appeared to be desirable.

\section{REFERENCES}

14. Charlton T. M. Energy principles in applied statics. London, Blackie, 1959, p. 38.

15. Szidarovszky J. Examination of accuracy of the Engesser-Vianello method and the Rayleigh quotient. Acta tech. Acad. Sci. Hung., 1962, 40 (1-2).

16. Asplund S. O. On deflexion theory of suspension bridges. Trans. sved Engng Acad., 1945, 184.

17. SzIDARovszky J. Producing influence lines of suspension bridges with variable flexural rigidity by successive approximation. Acta tech. Acad. Sci. Hung., 1962, $41(1-2)$. 